\title{
Compact Finite Differences Method for FitzHugh-Nagumo Equation
}

\author{
Canan Akkoyunlu ${ }^{1}$ \\ ${ }^{1}$ Department of Mathematics and Computer Science, Faculty of Science and Letters, Istanbul Kültür University, Istanbul, Turkey
}

\author{
Article Info \\ Keywords: FitzHugh-Nagumo equa- \\ tion, compact finite differences method. \\ 2010 AMS: $65 N 99$ \\ Received: 8 May 2019 \\ Accepted: 15 September 2019 \\ Available online: 26 December 2019
}

\begin{abstract}
In this paper, we developed the compact finite differences method to find approximate solutions for the FitzHugh-Nagumo (F-N) equations. To the best of our knowledge, until now there is no compact finite difference solutions have been reported for the FitzHughNagumo equation arising in gene propagation and model. We have given numerical example to demonstrate the validity and applicability.
\end{abstract}

\section{Introduction}

Nonlinear systems play a pivotal role in the mathematical modeling of scientific and engineering problems. The FitzHugh-Nagumo equation is a nonlinear reaction-diffusion equation that models an active pulse transmission line simulating a nerve axon [9] and it is used area of population genetics [4], circuit theory, and other fields [1], [8]. It is expressed as

$$
u_{t}-u_{x x}+u(1-u)(\rho-u)=0
$$

where $0<\rho<1$ and $u(x, t)$ is of the unknown function depending on the temporal variable $t$ and the spatial variable $x$. If we take $\rho=-1$, then Eq. (1.1) converts into the Newell-Whitehead equation.

$$
u_{t}-u_{x x}+u^{3}-u=0
$$

F-N equation (1.1) combines diffusion, and nonlinearity which is controlled by the term $u(1-u)(\rho-u)$. Many physicists and mathematicians have paid much attention to the Fitzhugh-Nagumo equation in recent years due to its importance in mathematical physics. Shih et al.[10] studied this equation and showed its applications in the field of population and circuit theory. In a study, the authors of a paper [6] examined the F-N equation and derived a novel series of exact solutions with the aid of the first integral technique. In another investigation, Abbasbandy [15] applied the homotopy anlaysis approach to obtain the soliton solution of the F-N equation. In an attempt, the authors of an article [13] investigated the variational method for solving both the Nagumo telegraph and the Nagumo reaction-diffusion partial differential equations. Jacobi elliptic function has been presented by Nucci and Clarkson [11] to obtain the solution of the F-N equation. In another study, Jackson [3] examined the semi-discrete estimates for the F-N equations. Moreover, the semi-analytical techniques have been successfully applied by Dehghan et al.[9], to present the approximate solution of the standard F-N equation.

In this work, we aim to investigate a generalized Fitzhugh-Nagumo equation with time-dependent coefficients and linear dispersion term given by

$$
u_{t}+\alpha(t) u_{x}-\beta(t) u_{x x}+\gamma(t) u(1-u)(\rho-u)=0, \quad(x, t) \in[A, B] \times[0, T]
$$

boundary condition

$$
u(A, t)=g_{1}(t), \quad u(B, t)=g_{2}(t), \quad t \in[0, T]
$$


initial condition

$$
u(x, 0)=f(t), \quad x \in[A, B]
$$

where $\alpha(t), \beta(t)$ and $\gamma(t)$ are arbitrary functions of t. $\alpha(t), \beta(t)$ and $\gamma(t)$ are all real-valued functions. For $\alpha(t)=0$ and $\beta(t)=\gamma(t)=1$, Eq. (1.2) will be reduced to the standard Fitzhugh-Nagumo equation (1.1).

The time-dependent Fitzhugh-Nagumo equation cannot be integrated by the classical integration methods. Triki and Wazwaz [7] examined a generalized F-N equation exhibiting time varying coefficients and linear dispersion term. Jacobi-Gauss-Lobatto collocation method has been applied for generalized F-N equation by Bhrawy [2].

Compact finite difference methods are techniques used in applied mathematics and scientific computing to numerically solve linear and nonlinear differential equations. Mohanty et al. [14] used new two-level implicit compact operator method for the solution of Burgers-Huxley equation. In a study, the authors of a paper [5] derived solution of the parabolic problems with delay using compact finite difference methods. Wang et al. [17] applied compact finite difference scheme to study the coupled Gross-Pitaevskii equations. In another investigation, Wu and $\mathrm{Xu}$ [18] derived the solutions of 2D Helmholtz equation with the compact sixth-order finite difference scheme.

Although there are many methods to construct the compact schemes, Pade Appoximation Method and Taylor Series Method which are the two basic approximations came into prominence. Many researchers are using higher order compact finite difference schemes to solve differential equations. This is because significant improvements to the accuracy of numerical solutions have been obtained by using fourth or sixth order compact finite difference schemes. Another advantage is that the high accuracy is obtained on coarser grids which ensures greater computational efficiency [12]. In this study, compact finite differences schemes for the first and second derivative approximations are constructed both for the inner points and the boundary points by using the Taylor approximation. Along the spatial coordinate, first and second derivatives are replaced with the fifth order compact schemes for the inner points and the sixth order compact schemes for the boundary points. The paper is arranged as follows: In Section 2, compact finite difference method for F-N equation is presented. In Section 3 , numerical results for different problems are presented in tables and conclusion is given in Section 4.

\section{Compact Finite Differences Method}

Compact finite difference method is a special finite difference method which uses the values of the function and its derivatives only at three consecutive points. The independent variable nodes are given as $x_{i}=h(i-1)$ where $h=(b-a) /(N-1)$, in the interval of $[a, b]$ for $1 \leq i \leq N$. If the function values at the nodes are given as $f_{i}=f\left(x_{i}\right)$ the finite differences approximation to the first derivative $f_{i}^{\prime}$ at the node indexed by $i$ depends on the function values at the neighbor nodes [16].

The approximation for the first derivative of the function is expressed as in the following

$$
f_{i-2}^{\prime}+\eta f_{i-1}^{\prime}+f_{i}^{\prime}+\eta f_{i+1}^{\prime}+\gamma f_{i+2}^{\prime}=c \frac{f_{i+3}-f_{i-3}}{6 h}+b \frac{f_{i+2}-f_{i-2}}{4 h}+a \frac{f_{i+1}-f_{i-1}}{2 h}
$$

where $f_{i}=f\left(x_{i}\right)$ and coefficients can be determined by the Taylor expansion. To get the fourth order tridiagonal schemes, the coefficients $\gamma$ and $c$ are set to be 0 . The order of accuracy for the approximations for the first and second derivatives are calculated as $O\left(h^{5}\right)$ for inner points and $O\left(h^{6}\right)$ for the boundary points. The approximation for the second derivative of the function is expressed as in the following

$$
\gamma f_{i-2}^{\prime \prime}+\eta f_{i-1}^{\prime \prime}+f_{i}^{\prime \prime}+\eta f_{i+1}^{\prime \prime}+\gamma f_{i+2}^{\prime \prime}=c \frac{f_{i+3}-2 f_{i}+f_{i-3}}{9 h^{2}}+b \frac{f_{i+2}-2 f_{i}+f_{i-2}}{4 h^{2}}+a \frac{f_{i+1}-2 f_{i}+f_{i-1}}{h^{2}}
$$

For boundary points, the approximation is written in the following form

$$
\begin{aligned}
f_{1}^{\prime}+\eta f_{2}^{\prime} & =\frac{1}{h}\left(a f_{1}+b f_{2}+c f_{3}+d f_{4}\right) \\
f_{1}^{\prime \prime}+\eta f_{2}^{\prime \prime} & =\frac{1}{h^{2}}\left(a f_{1}+b f_{2}+c f_{3}+d f_{4}+e f_{5}\right)
\end{aligned}
$$

The equations (2.1) and (2.2) can easily be adapted for the boundary point $i=N$. The compact schemes for the first and second derivative approximations for the interior and boundary points with their calculated coefficients can be seen below.

For the first derivative

$$
\begin{aligned}
\frac{h}{3}\left(-17 u_{1}^{\prime}-14 u_{2}^{\prime}+u_{3}^{\prime}\right) & =u_{0}+8 u_{1}-9 u_{2} \\
\frac{h}{3}\left(u_{i-1}^{\prime}+4 u_{i}^{\prime}+u_{i+1}^{\prime}\right) & =-u_{i-1}+u_{i+1} \\
\frac{h}{3}\left(\frac{1}{8} u_{N-3}^{\prime}-\frac{5}{8} u_{N-2}^{\prime}+\frac{19}{8} u_{N-1}^{\prime}+\frac{9}{8} u_{N}^{\prime}\right) & =-u_{N-1}+u_{N}
\end{aligned}
$$

For the second derivative

$$
\begin{aligned}
\frac{h^{2}}{12}\left(14 u_{1}^{\prime \prime}-5 u_{2}^{\prime \prime}+4 u_{3}^{\prime \prime}-5 u_{4}^{\prime \prime}\right) & =u_{0}-2 u_{i}+u_{i+1} \\
\frac{h^{2}}{12}\left(u_{i-1}^{\prime \prime}+10 u_{i}^{\prime \prime}+u_{i+1}^{\prime \prime}\right) & =u_{i-1}-2 u_{i}+u_{i+1} \\
\frac{h^{2}}{12}\left(-u_{N-4}^{\prime \prime}+4 u_{N-3}^{\prime \prime}-5 u_{N-2}^{\prime \prime}+14 u_{N-1}^{\prime \prime}\right) & =u_{N-2}-2 u_{N-1}+u_{N}
\end{aligned}
$$


By taking 7 nodes, the matrices obtained from (2.3), (2.4) and (2.5) are as in the following.

$$
\begin{aligned}
& A_{1}=\left(\begin{array}{ccccc}
-\frac{17 h}{3} & -\frac{14 h}{3} & \frac{h}{3} & 0 & 0 \\
\frac{h}{3} & \frac{4 h}{3} & \frac{h}{3} & 0 & 0 \\
0 & \frac{h}{3} & \frac{4 h}{3} & \frac{h}{3} & 0 \\
0 & 0 & \frac{h}{3} & \frac{4 h}{3} & \frac{h}{3} \\
0 & \frac{h}{24} & \frac{-5 h}{24} & \frac{19 h}{24} & \frac{9 h}{24}
\end{array}\right), \quad U^{\prime}=\left(\begin{array}{c}
u_{1}^{\prime} \\
u_{2}^{\prime} \\
u_{3}^{\prime} \\
u_{4}^{\prime} \\
u_{5}^{\prime}
\end{array}\right) \\
& K_{1}=\left(\begin{array}{ccccc}
8 & -9 & 0 & 0 & 0 \\
-1 & 0 & 1 & 0 & 0 \\
0 & -1 & 0 & 1 & 0 \\
0 & 0 & -1 & 0 & 1 \\
0 & 0 & 0 & -1 & 1
\end{array}\right), \quad H_{1}=\left(\begin{array}{c}
u_{0} \\
0 \\
0 \\
0 \\
0
\end{array}\right)
\end{aligned}
$$

The matrix $U^{\prime}$ having the approximation values of the first derivative at each node is calculated via the equation (2.9). The following calculations are carried out to obtain the matrix $U^{\prime}$ after applying the $L U$ decomposition technique to the known matrix $A_{1}$

$$
A_{1} U^{\prime}=K_{1} U+H_{1}
$$

$L U$ decomposition technique is applied to the known matrix $A_{1}$ and other calculations are as in the following.

$$
\begin{aligned}
\left(L_{0} U_{0}\right) U^{\prime} & =K_{1} U+H_{1} \\
L_{0}^{-1} L_{0} U_{0} U^{\prime} & =L_{0}^{-1} K_{1} U+L_{0}^{-1} H_{1} \\
T_{1} & =L_{0}^{-1} K_{1} \\
G_{1} & =L_{0}^{-1} H_{1} \\
U_{0} U^{\prime} & =T_{1} U+G_{1} \\
U_{0}^{-1} U_{0} U^{\prime} & =U_{0}^{-1} T_{1} U+U_{0}^{-1} G_{1} \\
S_{1} & =U_{0}^{-1} G_{1} \\
C_{1} & =U_{0}^{-1} T_{1} \\
U^{\prime}=C_{1} U & \\
&
\end{aligned}
$$

Using the compact schemes in (2.6), (2.7) and (2.8), the matrices below are obtained to get the matrix $U^{\prime \prime}$ having the approximation values of the second derivative at each node and similar calculations are carried out for that. $U^{\prime}$ and $U^{\prime \prime}$ are inserted as a first and second derivatives of the function while constructing the discretization scheme.

$$
\begin{aligned}
& A_{2}=\left(\begin{array}{ccccc}
\frac{14 h^{2}}{12} & -\frac{5 h^{2}}{12} & \frac{4 h^{2}}{12} & -\frac{h^{2}}{12} & 0 \\
\frac{h^{2}}{12} & \frac{10 h^{2}}{12} & \frac{h^{2}}{12} & 0 & 0 \\
0 & \frac{h^{2}}{12} & \frac{10 h^{2}}{12} & \frac{h^{2}}{12} & 0 \\
0 & 0 & \frac{h^{2}}{12} & \frac{10 h^{2}}{12} & \frac{h^{2}}{12} \\
0 & -\frac{h^{2}}{12} & \frac{4 h^{2}}{12} & -\frac{5 h^{2}}{12} & \frac{14 h^{2}}{12}
\end{array}\right), \quad U^{\prime \prime}=\left(\begin{array}{c}
u_{1}^{\prime \prime} \\
u_{2}^{\prime \prime} \\
u_{3}^{\prime \prime} \\
u_{4}^{\prime \prime} \\
u_{5}^{\prime \prime}
\end{array}\right) \\
& U=\left(\begin{array}{c}
u_{1} \\
u_{2} \\
u_{3} \\
u_{4} \\
u_{5}
\end{array}\right), \quad K_{2}=\left(\begin{array}{ccccc}
-2 & 1 & 0 & 0 & 0 \\
1 & -2 & 1 & 0 & 0 \\
0 & 1 & -2 & 1 & 0 \\
0 & 0 & 1 & -2 & 1 \\
0 & 0 & 0 & 1 & -2
\end{array}\right), H_{2}=\left(\begin{array}{c}
u_{0} \\
0 \\
0 \\
0 \\
u_{6}
\end{array}\right) \\
& A_{2} U^{\prime \prime}=K_{2} U+H_{2} \\
& U^{\prime \prime}=C_{2} U+S_{2}
\end{aligned}
$$

\subsection{Compact Finite Difference method for Fitzhugh-Nagumo equation}

Equations (2.10) and (2.11) approximating to the first and second derivatives of functions $u$ is substituted to the equation (1.2). Compact finite differences for spatial dimension and finite differences along the time axis are applied, consequently they are rearranged via explicit approximation and the following discretization scheme is obtained.

$$
\frac{U_{n}^{j+1}-U_{n}^{j}}{\Delta t}+\alpha\left(t_{j}\right)\left(C_{1} U_{n}^{j}+S_{1}\right)-\beta\left(t_{j}\right)\left(C_{2} U_{n}^{j}+S_{2}\right)+\gamma\left(t_{j}\right) U_{n}^{j}\left(1-U_{n}^{j}\right)\left(\rho-U_{n}^{j}\right)
$$


While the approximations to the functions $u$ via compact scheme is being constructed, the known boundary values are placed in the vectors $H_{1}$ and $H_{2}$. And the vectors $S_{1}$ and $S_{2}$ are calculated in each time step. Because they are dependent on the approximation values of $u$ after the first step.

$$
H_{1}=\left(\begin{array}{c}
u_{0} \\
0 \\
0 \\
0 \\
0
\end{array}\right), \quad H_{2}=\left(\begin{array}{c}
u_{0} \\
0 \\
0 \\
0 \\
u_{N}
\end{array}\right)
$$

\section{Numerical Results}

In this section, solution for equation in (1.2) is obtained via compact finite differences method. To illustrate the efficiency of the compact finite differences method for the problem handled in this study, the maximum error which is defined by the equation below

$$
L_{\infty}=\max _{1 \leq j \leq N}\left|u\left(x_{j}, t\right)-U\left(x_{j}, t\right)\right|
$$

where $u\left(x_{j}, t\right)$ and $U\left(x_{j}, t\right)$ refer to the exact solution and solution via compact finite differences method, respectively.

Example 1. Consider equation (1.2) with $\alpha(t)=0, \beta(t)=1, \gamma(t)=1$ :

$$
u_{t}-u_{x x}+u(1-u)(\rho-u)=0 ; \quad(x, t) \in[-10,10] \times[0, T]
$$

subject to the boundary conditions

$$
\begin{gathered}
u(-10, t)=\frac{1}{2}+\frac{1}{2} \tanh \left(\frac{1}{2 \sqrt{2}}\left(-10-\frac{2 \rho-1}{\sqrt{2}} t\right)\right) \\
u(10, t)=\frac{1}{2}+\frac{1}{2} \tanh \left(\frac{1}{2 \sqrt{2}}\left(10-\frac{2 \rho-1}{\sqrt{2}} t\right)\right)
\end{gathered}
$$

and the initial condition

$$
u(x, 0)=\frac{1}{2}+\frac{1}{2} \tanh \left(\frac{x}{2 \sqrt{2}}\right), x \in[-10,10]
$$

The exact solution of Eq. (3.1) is

$$
u(x, t)=\frac{1}{2}+\frac{1}{2} \tanh \left(\frac{1}{2 \sqrt{2}}\left(x-\frac{2 \rho-1}{\sqrt{2}} t\right)\right)
$$

The results for example 1 are displayed in Tables 4.1 and Figure 3.2. Numerical solutions at different $T$ are presented for computational domain $[-10,10]$ using compact finite difference method in Table 1. And in Table 2, we exhibit the maximum absolute errors between exact and approximate solutions for $N=12,48,64$. The Fig. 3.1 display the numerical results with exact one for $\rho=0.75, \Delta t=0.001, N=24$ in 3D form up to time $T=1$. The Fig. 3.2 compares the numerical solutions with exact one for different $\rho$ values. As it can be seen from the Table 1 and Table 2 the compact finite differences method is very accurate. 

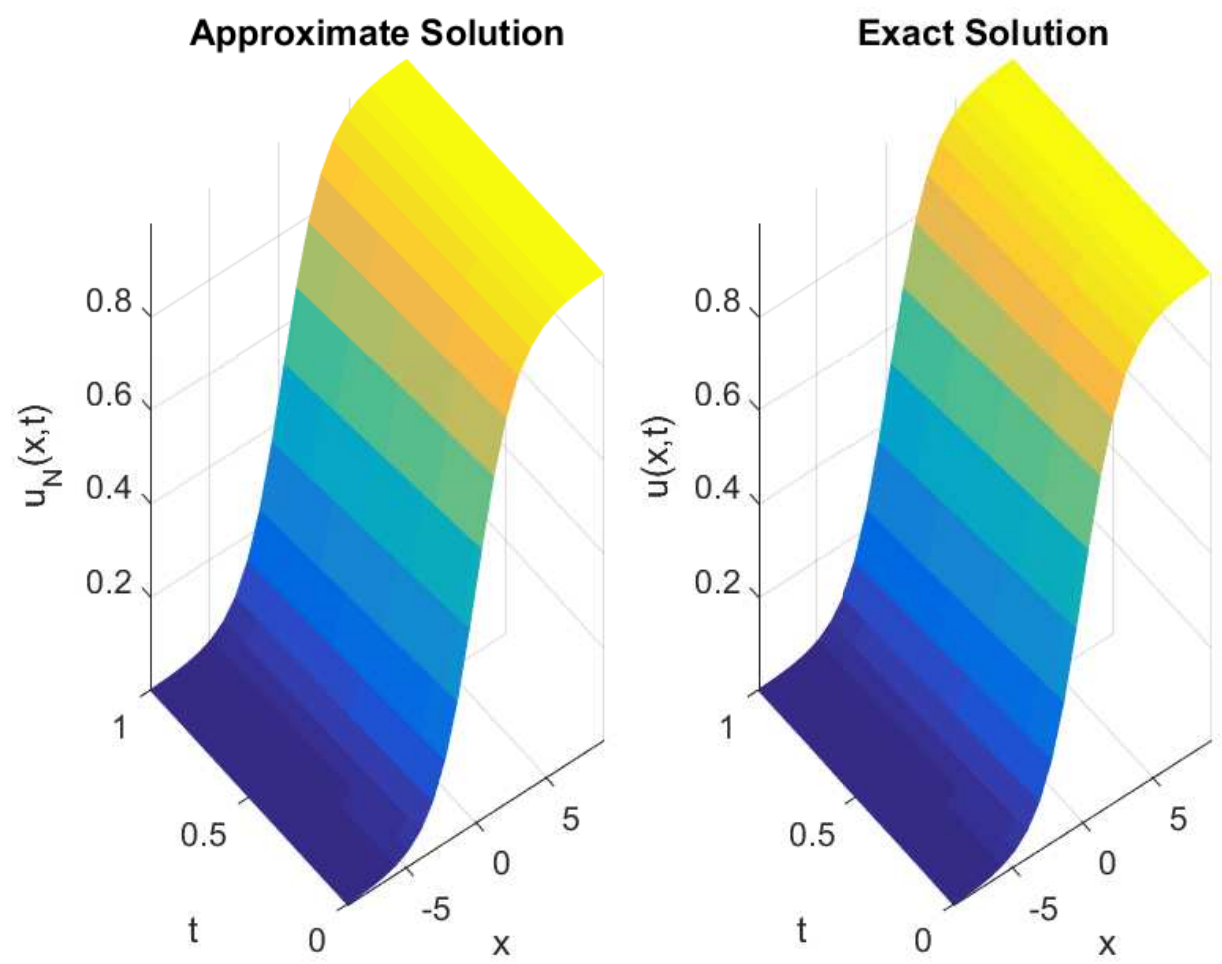

Figure 3.1: For $N=24, \Delta t=0.001, T=1, \rho=0.75$ solution of Fitzhugh-Nagumo equation for Example 1

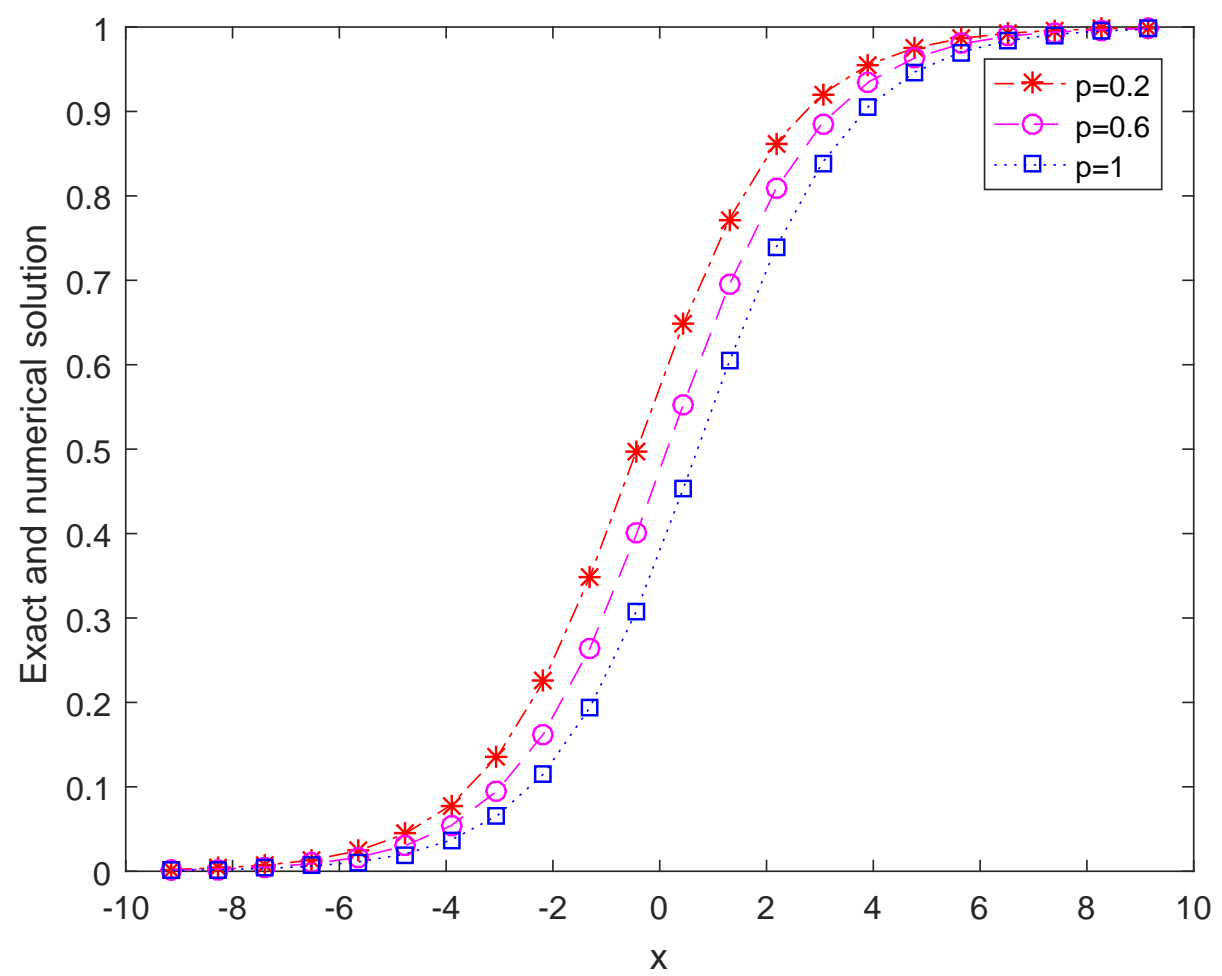

Figure 3.2: For $N=24, \Delta t=0.001, T=1$ solution of Fitzhugh-Nagumo equation for Example 1

Table 2:

Example 2. In this example we examine the nonlinear time-dependent generalized F-H equation with time coefficients.

$$
u_{t}+\cos (t) u_{x}-\cos (t) u_{x x}-2 \cos (t)(u(1-u)(\rho-u))=0 ;(x, t) \in[-10,10] \times[0, T]
$$




\begin{tabular}{cccccc}
\hline $\mathrm{dt}$ & $\mathrm{T}=0.2$ & $\mathrm{~T}=1$ & $\mathrm{~T}=2$ & $\mathrm{~T}=3$ & $\mathrm{~T}=4$ \\
\hline $\mathrm{dt}=0.1$ & $7.2090 \mathrm{e}-05$ & $2.8991 \mathrm{e}-04$ & $5.2054 \mathrm{e}-04$ & $6.8524 \mathrm{e}-04$ & $8.8408 \mathrm{e}-04$ \\
$\mathrm{dt}=0.01$ & $2.3475 \mathrm{e}-05$ & $8.0706 \mathrm{e}-05$ & $1.1019 \mathrm{e}-04$ & $1.5188 \mathrm{e}-04$ & $1.7331 \mathrm{e}-04$ \\
$\mathrm{dt}=0.001$ & $1.8739 \mathrm{e}-05$ & $6.2242 \mathrm{e}-05$ & $7.7129 \mathrm{e}-05$ & $9.8812 \mathrm{e}-05$ & $1.1203 \mathrm{e}-04$ \\
$\mathrm{dt}=0.0001$ & $1.8267 \mathrm{e}-05$ & $6.0398 \mathrm{e}-05$ & $7.4378 \mathrm{e}-05$ & $9.3508 \mathrm{e}-05$ & $1.0590 \mathrm{e}-04$
\end{tabular}

Table 1: Maximum error with $\rho=0.75$ and $N=24$ for Example 1

\begin{tabular}{cccc}
\hline $\mathrm{N}$ & $\mathrm{dt}=0.01$ & $\mathrm{dt}=0.001$ & $\mathrm{dt}=0.0001$ \\
\hline 12 & $3.9857 \mathrm{e}-04$ & $3.9300 \mathrm{e}-04$ & $3.9244 \mathrm{e}-04$ \\
48 & $8.3749 \mathrm{e}-06$ & $4.0905 \mathrm{e}-06$ & $3.7343 \mathrm{e}-06$ \\
64 & $5.9363 \mathrm{e}-06$ & $8.1794 \mathrm{e}-07$ & $3.9098 \mathrm{e}-07$
\end{tabular}

Table 2: Maximum error with $T=0.2$ and $\rho=0.75$ for Example 1

subject to the boundary conditions

$$
\begin{aligned}
u(-10, t) & =\frac{\rho}{2}+\frac{\rho}{2} \tanh \left(\frac{\rho}{2}(-10-(3-\rho) \sin (t))\right), \\
u(10, t) & =\frac{\rho}{2}+\frac{\rho}{2} \tanh \left(\frac{\rho}{2}(10-(3-\rho) \sin (t))\right)
\end{aligned}
$$

and initial condition

$$
u(x, 0)=\frac{\rho}{2}+\frac{\rho}{2} \tanh \left(\frac{\rho x}{2}\right), x \in[-10,10]
$$

The analytical solution of Eq.(3.2) is

$$
u(x, t)=\frac{\rho}{2}+\frac{\rho}{2} \tanh \left(\frac{\rho}{2}(x-(3-\rho) \sin (t))\right)
$$

The observed maximum absolute errors for different values of $\rho$ are given in Table 3. The numerical results are illustrated in Fig. 3.3

\section{Approximate Solution}

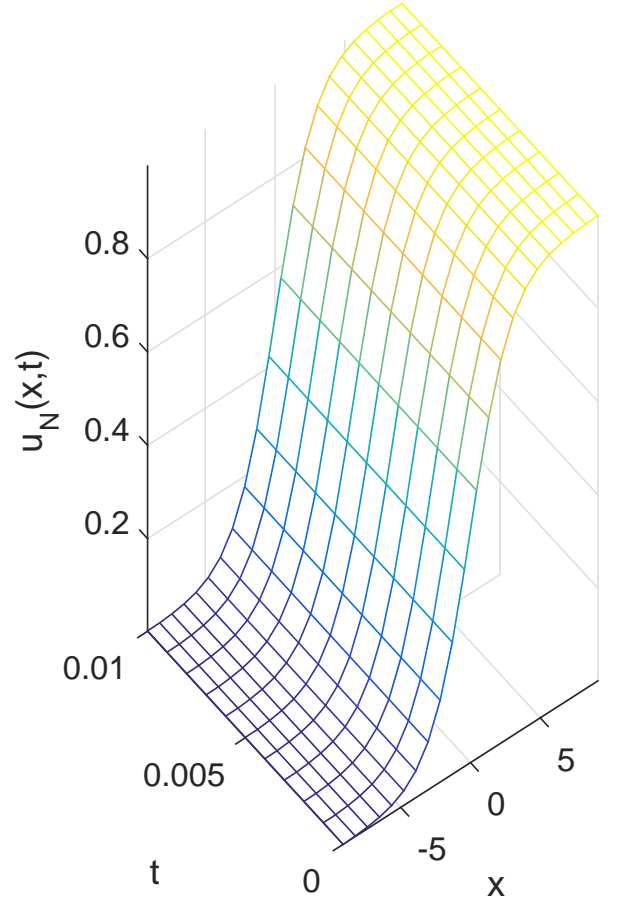

Exact Solution

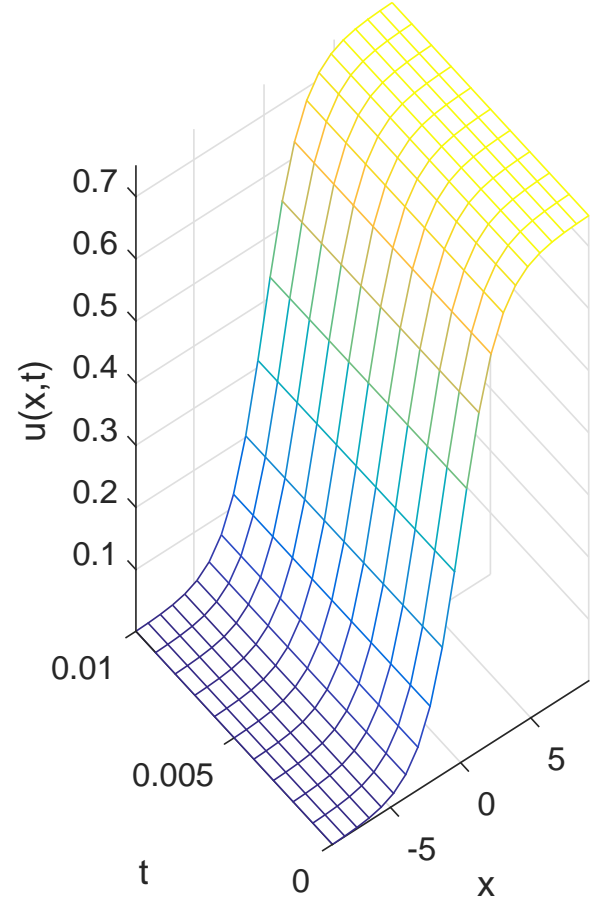

Figure 3.3: For $N=24, \Delta t=0.001, T=0.01, \rho=0.75$, solution of Fitzhugh-Nagumo equation for Example 2 


\begin{tabular}{cccc}
\hline$\rho$ & $\mathrm{dt}=0.1$ & $\mathrm{dt}=0.01$ & $\mathrm{dt}=0.001$ \\
\hline$\rho=0.25$ & 0.0449 & 0.0451 & 0.0451 \\
$\rho=0.5$ & 0.1481 & 0.1489 & 0.1490 \\
$\rho=0.75$ & 0.2682 & 0.2700 & 0.2701
\end{tabular}

Table 3: Maximum error with $T=1$ and $N=24$ for Example 2
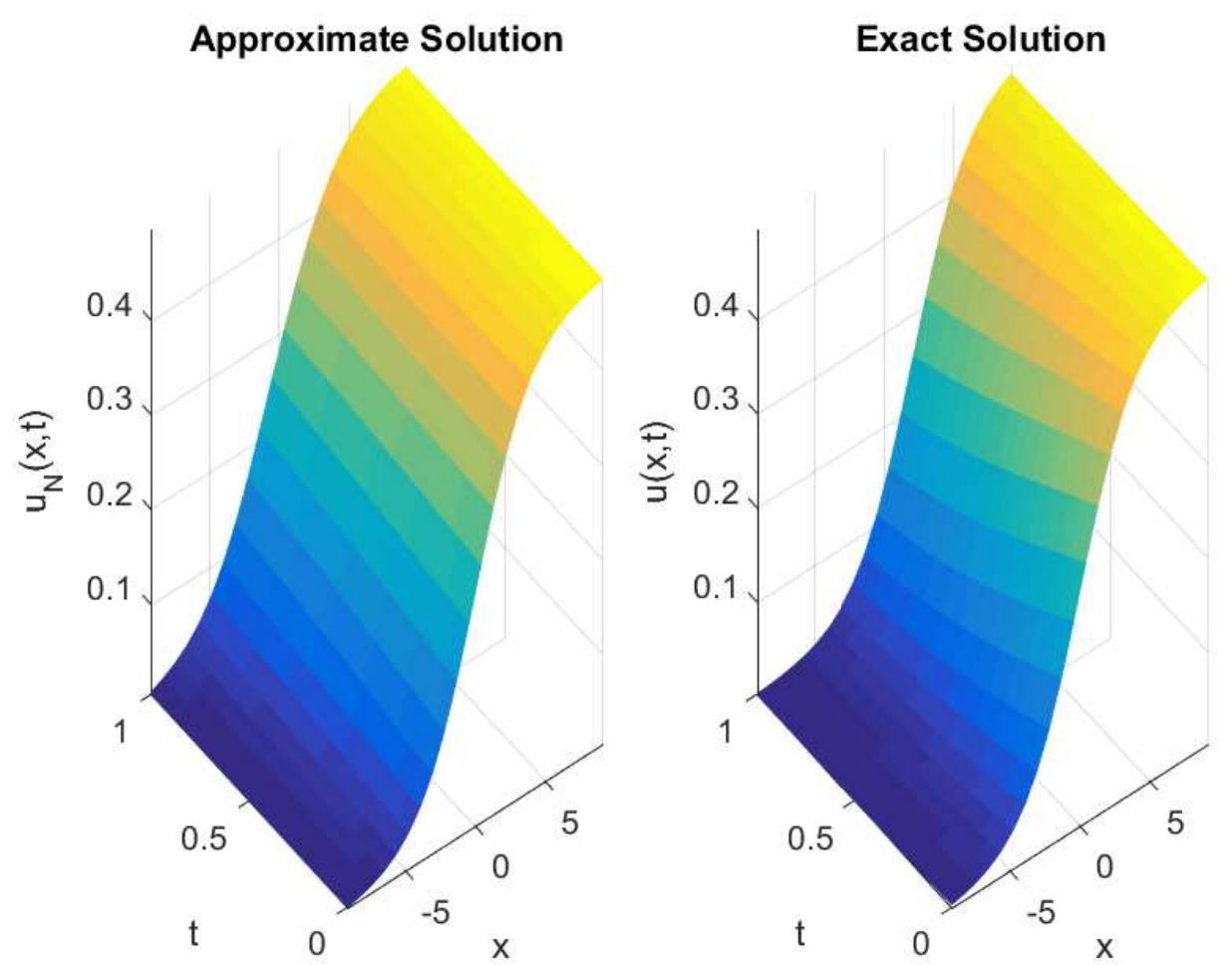

Figure 3.4: For $N=24, \Delta t=0.001, T=0.01, \rho=0.05$, solution of Fitzhugh-Nagumo equation for Example 2

\section{Conclusion}

In this study, compact finite differences method is used to solve Fitzhugh-Nagumo equation. To check the accuracy of the method two test problems available in the literature are solved. The maximum absolute errors are presented in Tables with different time step. From examples, we have observed that the choice of particular $\rho$ values can affect accurate of the numerical solutions. It is suggested that the compact finite different method produced accurate solution.

\section{References}

[1] A. C. Scott, Neunstor propagation on a tunnel diode loaded transmission line, Proceedings of IEEE 51 (1963), $240-249$.

[2] A. H. Bhrawy, A Jacobi-Gauss-Lobatto collocation method for solving generalized Fitzhugh-Nagumo equation with time-dependent coefficients, Appl. Math. Comput., 222 (2013), 255-264.

[3] D. E. Jackson, Error estimates for the semidiscrete Galerkin approximations of the Fitzhugh-Nagumo equations, Appl. Math. Comput., 50 (1992), 93-114.

[4] D. G. Aronson, H. F. Weinberger, Multidimensional nonlinear diffusion arising in population genetics, Advances in Mathematics, 30 (1978), $33-76$.

[5] F. Wu, D. Li, J. Wen, J. Duan, Stability and convergence of compact finite difference method for parabolic problems with delay, Appl. Math. and Comp., 322 (2018), 129-139.

[6] H. Li, Y. Guo, New exact solutions to the Fitzhugh-Nagumo equation, Appl. Math. Comput., 180 (2006), $524-528$.

[7] H. Triki, A.-M. Wazwaz, On soliton solutions for the Fitzhugh-Nagumo equation with time-dependent coefficients, Appl. Math. Model., 37 (2013) 3821-3828.

[8] J. Nagumo, S. Yoshizawa, S. Arimoto, Bistable trunsmission lines, Transactions on IEEE Circuit Theory, 12 (1965) 400-412.

[9] M. Dehghan, J. M. Heris, A. Saadatmandi, Application of semi-analytic methods for the Fitzhugh-Nagumo equation, which models the transmission of nerve impulses, Math. Methods Appl. Sci., (2010)

[10] M. Shih, E. Momoniat, F. M. Mahomed, Approximate conditional symmetries and approximate solutions of the perturbed Fitzhugh-Nagumo equation, J. Math. Phys., 46 (2005), (023503).

[11] M. C. Nucci, P. A. Clarkson, The nonclassical method is more general than the direct method for symmetry reductions: an example of the FitzhughNagumo equation, Phys Lett. A, 164 (1992), 49-56.

[12] P. G. Dlamini and M. Khumalo, A new compact finite difference quasilinearization method for nonlinear evolution partial differential equations, Open Math., 15 (2017), 1450-1462.

[13] R. A. Van Gorder, A variational formulation of the Ngumo reaction-diffusion equation and the Nagumo telegraph equation, Nonlinear Anal. Real World Appl., 11 (2010), 2957-2962. 
[14] RK. Mohanty, D. Weizhong, L. Donn, Operator compact method of accuracy two time in time and four in space for the solution of time dependent Burgers-Huxley equation, Numer Algor, 70 (2015), 591-605.

[15] S. Abbasbandy, Soliton solutions for the Fitzhugh-Nagumo equation with the homotopy analysis method, Appl. Math. Model., 32 (2008), 2706-2714.

[16] SK. Lele, Compact finite difference schemes with Spectral-like Resolution, Journal of Computational Physics, 103 (1992), 16-42.

[17] T. Wang, J. Jiang, H. Wang, W. Xu, An efficient and conservative compact finite difference scheme for the coupled Gross-Pitaevskii equations describing

spin-1 Bose-Einstein condensate, Appl. Math. and Comp., 323 (2018), 164-181.
[18] T. Wu, R. Xu, An optimal compact sixth-order finite difference scheme for the Helmholtz equation, Comp. Math. and Appl., (2018). 